\title{
Towards Migrating Computation to Distributed Memory Caches
}

\author{
Adam Schaub \\ Cisco \\ adamscha@cisco.com
}

\author{
Michael Spear \\ Lehigh University \\ spear@cse.lehigh.edu
}

\begin{abstract}
Memcached and other in-memory distributed key-value stores play a critical role in large-scale web applications, by reducing traffic to persistent storage and providing an easy-to-access look-aside cache in which programmers can store arbitrary data. These caches typically have a narrow interface, consisting only of gets, sets, and compare-and-set. In the worst case, this interface can cause significant inefficiencies as clients get large data items, perform small changes, and then set the updated items back into the cache.

We extend memcached to allow clients to execute code directly in the cache. An idealized evaluation on micro-benchmarks based on workload traces from a Cable/Internet service provider shows compelling performance, leading to a recommendation that further research be conducted to make in-cache fetch-and-phi safe and programmer-friendly, and that researchers consider whether a truly distributed cloud platform should make it easier for programmers to execute custom code at all levels of the software stack.
\end{abstract}

\section{CCS Concepts}

-Computing methodologies $\rightarrow$ Distributed programming languages; •Software and its engineering $\rightarrow$ Cloud computing;

\section{Keywords}

Fetch-and-phi, Key-value store

\section{INTRODUCTION}

Interactive distributed systems often rely on an in-memory cache of key/value pairs [1]. This cache can serve many roles, to include providing fast access to information from persistent table stores like HBase, BigTable [2], Silt [8], Cassandra, and Dynamo [3]; caching results from expensive joins in traditional relational databases [1]; and storing the result of expensive computations. Memcached [10] is one of the most popular and widely-used key/value caches.

There exist times when these caches can be a bottleneck. Our specific motivation in this paper is the infrastructure of Comcast's XFINITY X1 user interface. Millions of set-top boxes in a geographic market serve as thin clients, forwarding clicks from a cus-

Permission to make digital or hard copies of all or part of this work for personal or classroom use is granted without fee provided that copies are not made or distributed for profit or commercial advantage and that copies bear this notice and the full citation on the first page. Copyrights for components of this work owned by others than ACM must be honored. Abstracting with credit is permitted. To copy otherwise, or republish, to post on servers or to redistribute to lists, requires prior specific permission and/or a fee. Request permissions from permissions@ acm.org.

DCC'16, July 25-28, 2016, Chicago, IL, USA

(C) 2016 ACM. ISBN 978-1-4503-4220-9/16/07 . \$ \$15.00

DOI: http://dx.doi.org/10.1145/2955193.2955202 tomer's remote control to Comcast logic servers. In response to a click, a logic server will access memcached and/or Cassandra layers, and then render a new screen that is sent back to the customer. Unlike many bursty workloads, this system experiences predictable spikes in use. At fixed times (e.g., 9:00 PM), millions of Digital Video Recorders (DVRs) simultaneously stop one recording and begin another. Each logic server must, for each of its DVRs, start or stop a physical recording operation and also update the customer's UI metadata. The UI data is stored several ways: first, the individual recording is updated, and then the views of the recordings ("scheduled", "in progress", "completed") are updated. These bursts are too regular to be mitigated through sharing other servers, since the burst is periodic and relies on data being stored in-memory.

This workload exhibits a behavior that we believe generalizes: writes of a datum are preceded by a read of the same datum, and the portion of the data that is written is small. When objects are large (tens, if not hundreds of kilobytes), an opportunity emerges: if it were possible to update the object directly in the cache, and then return only the updated object, we could halve the number of network round trips, and halve the required bandwidth.

Our desired behavior is depicted in Figure 1. On the left side, there are two expensive communications, labeled 3 and 5, which correspond to getting a large object $O$ and setting the updated object $O^{\prime}$ back into the cache. The messages sent in steps 2 and 6 are small, representing a get request, and an (optional) acknowledgment. On the right hand side, when the computation (represented by a star) migrates to the cache server, one round trip network communication is avoided, and the only large object transmission occurs at step 4.

Our extensions to memcachedprovide an atomic fetch-and-phi primitive [6], which allows a programmer to get an object, invoke a function on that object (possibly involving programmer-specified parameters), and set the result back into the cache, all in a single atomic operation that returns the computed value. Variants of our implementation can elide the set and/or omit the response. Evaluation on micro-benchmarks and traces from Comcast's production servers shows that moving computation into the cache can reduce overheads and increase throughput by more than $30 \%$.

Though compelling, our result is not immediately usable: we had to rewrite portions of Comcast's logic in C to facilitate execution within the memcached process, raising questions about reliability and security. Additional issues, such as the data format (in our case, Google Protocol Buffers) and compression (which was disabled) can affect performance, by increasing server CPU utilization. We hope that in presenting our result, we can encourage further research in the operating systems, database, programming language, and distributed computing communities, to explore safe and 

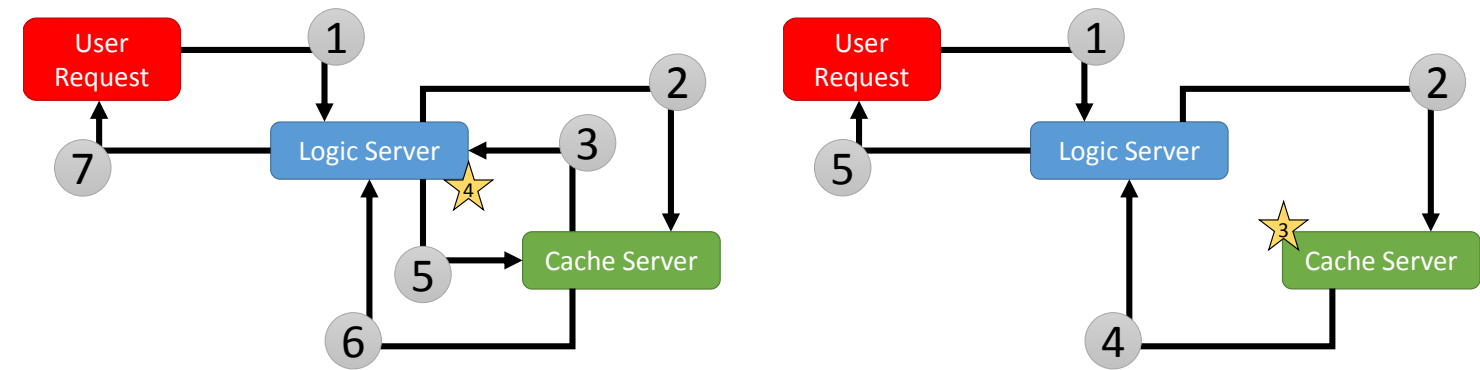

Figure 1: Default system behavior (left) and behavior with in-cache computation (right). Arrows represent the flow of information (requests and responses), and stars represent computation.

programmer-friendly migration of code, not only to cache servers, but throughout distributed clouds.

\section{EXTENDING MEMCACHED}

Table 1 presents the memcached API. Three functions comprise the primary API: get takes a key, and returns the corresponding object; set takes a key and value, and updates the value stored at that key; delete removes a key/value pair. Each key is stored with a version number that increments on every set, replace, append, prepend, incr, and delete. By using gets instead of get, a client can observe this value, and using cas instead of set achieves a read-modify-write, akin to load-linked/store conditional: cas takes a key, a value, and the counter value. It updates the value if and only if the counter values match, and updates the counter.

When the value is an integer, incr and decr can effect an atomic increment or decrement. Memcached effectively locks the key/value pair, updates the value, and then releases the lock. The same approach can be used when the value is treated as a raw byte array, and the operation is append or prepend. However, there is an additional constraint in this case: every object has a size class (slab) associated with it. Within the slab, each object is stored with some internal fragmentation, and a prepend or append will fail if the new object cannot fit in the object's existing slab. The set, add, replace, and cas operations are not subject to this restriction, since they assign the newly provided data to the key, instead of modifying the key in-place. To this API, we add two new operations: register() and invoke().

\subsection{Register and Invoke}

Register () performs inserts and lookups into a map of $<$ string, function $>$ pairs. The function returns a boolean, and takes the following parameters:

\begin{tabular}{lll} 
Name & Type & Purpose \\
\hline key & byte[] (in) & $\begin{array}{l}\text { The key with which this function was } \\
\text { called } \\
\text { The value most recently returned by } \\
\text { old_val }\end{array}$ \\
bytey)
\end{tabular}

By exploiting memcached's mechanism for coordinating resizes of the underlying hash table, the map can be accessed without synchronization: modifying the map, through the register () function, is a maintenance operation during which no client requests are active.

Invoke () executes a function that was previously registered on the server. It takes the following parameters:

\begin{tabular}{|c|c|}
\hline Command & Purpose \\
\hline set & $\begin{array}{l}\text { Store data, possibly overwriting the existing } \\
\text { value for the given key; promote the key to the } \\
\text { top of the LRU list. }\end{array}$ \\
\hline add & $\begin{array}{l}\text { Store data only if key does not exist; promote the } \\
\text { key to the top of the LRU list even if key already } \\
\text { in cache. }\end{array}$ \\
\hline replace & $\begin{array}{l}\text { Store key/value pair only if the key already exists } \\
\text { in the cache. }\end{array}$ \\
\hline append & $\begin{array}{l}\text { Extend an existing value by concatenating the } \\
\text { supplied data to the end. Constrained by pre- } \\
\text { determined size class of a value. }\end{array}$ \\
\hline prepend & $\begin{array}{l}\text { Extend an existing value by concatenating to the } \\
\text { front of the value. Same constraints as append. }\end{array}$ \\
\hline cas & $\begin{array}{l}\text { Compare-and-swap: performs a replace only if } \\
\text { the value hasn't changed since the last gets of } \\
\text { the key by the client. }\end{array}$ \\
\hline get & Retrieve and return data. \\
\hline gets & $\begin{array}{l}\text { Perform get, and return additional metadata for } \\
\text { use in a cas. }\end{array}$ \\
\hline delete & Remove an item from the cache, if it exists. \\
\hline incr & $\begin{array}{l}\text { Add to the value of a key only if the key is an } \\
\text { integer. }\end{array}$ \\
\hline decr & $\begin{array}{l}\text { Subtract from the value of a key only if the key } \\
\text { is an integer. }\end{array}$ \\
\hline stats $[\mathrm{p}]$ & $\begin{array}{l}\text { Interact with statistics information. Uses } \mathrm{p} \text { to re- } \\
\text { quest detailed statistics about items or slabs. }\end{array}$ \\
\hline
\end{tabular}

Table 1: The memcached client API. For clarity, we ignore that some functions can take multiple keys.

\begin{tabular}{lll} 
Name & Type & Purpose \\
\hline $\begin{array}{l}\text { name } \\
\text { key }\end{array}$ & $\begin{array}{l}\text { String (in) } \\
\text { byte[] (in) }\end{array}$ & $\begin{array}{l}\text { The name of the function to call } \\
\text { The key whose value will be used by } \\
\text { the function }\end{array}$ \\
params & byte[] (in) & $\begin{array}{l}\text { Parameters to pass to the function } \\
\text { being called }\end{array}$ \\
update & Boolean (in) & $\begin{array}{l}\text { True if the value should be updated } \\
\text { reply }\end{array}$ \\
The API makes no assumptions about how invoked functions are
\end{tabular}
used. The boolean parameters determine if if the function is a filter (the reply is not written back to the cache), an atomic fetch-andphi, or an atomic fetch-and-phi without a response. Algorithm 1 presents the pseudocode for invoke(). The client issues an invoke by supplying the name of a function, a key, a parameter string, and a few flags.

The operation consists of five stages. The first stage (lines 2-5) entails locating the code that will be executed, via a lookup in the map. Stage 2 (lines 6-9) fetches the data currently associated with the key, along with its current version count. Stage 3 (lines 10- 


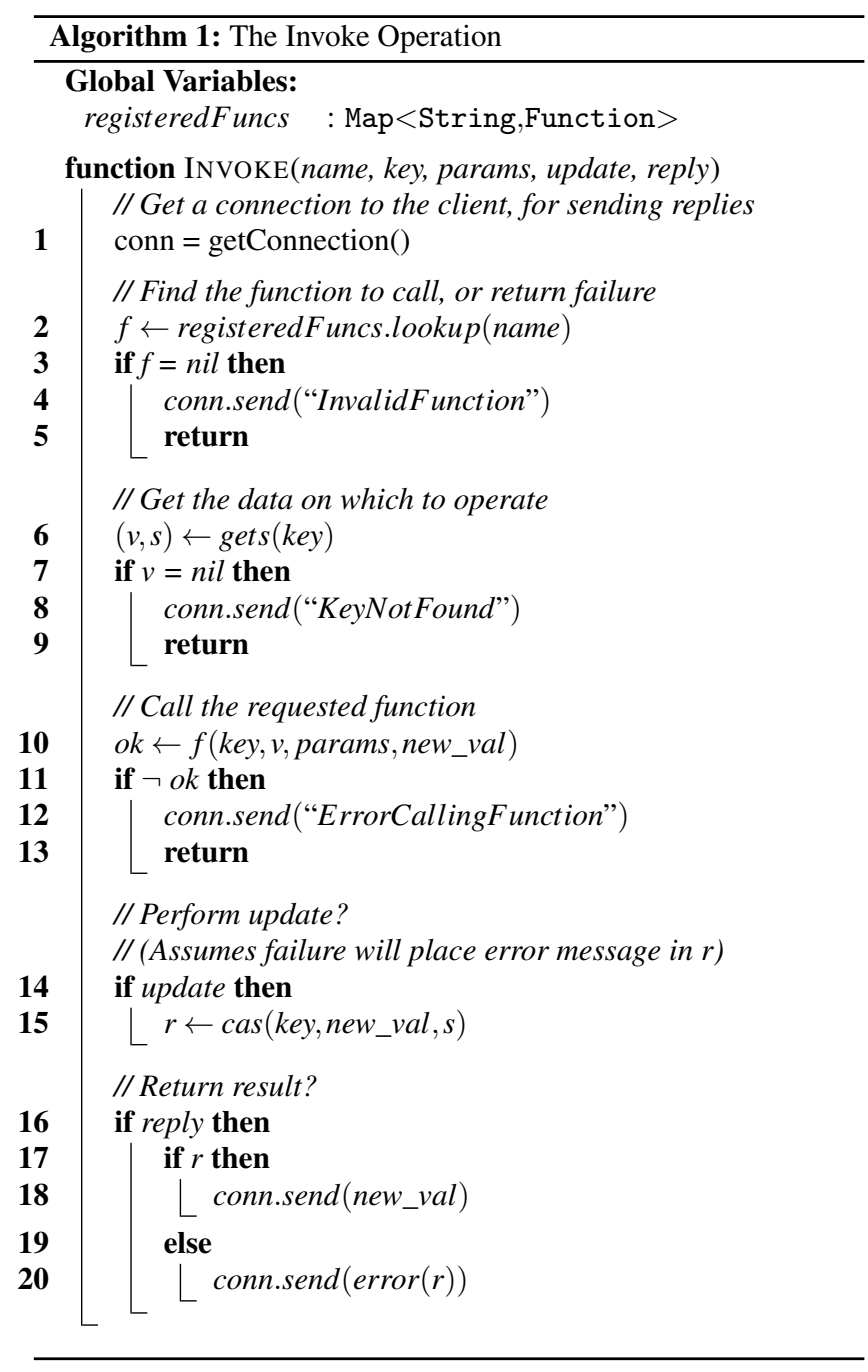

13) involves calling the requested function, passing the key, current value, and parameters. This produces a status message (success/failure) as well as a new byte array. This byte array may be a new version of the value, or some computed value of some other type. The meaning of the contents of the new_val parameter are programmer-defined. Stage 4 (lines 14-15) is to (optionally) update memcached so that the new value is associated with the key. For brevity, we omit details of how errors at this point are relayed to the client. Stage 5 (lines 16-20) returns the result of the function to the client.

\subsection{Generality and Reliability}

While our focus is on reducing overhead for a fetch-and-phi pattern that replaces a get-then-set, invoke() is general enough to support two additional usage patterns. First, we support filtering, which essentially mirrors a SELECT query: if we think of the value in the cache as a recordset, then the invoke can return a subset of the bytes of the recordset. This can minimize network traffic for lookups of small fields of big objects. Second, we allow filtering with computation, where the invoked function processes the data and returns a result. This is beneficial when many fields of a large object are used, but only a small amount of data is output by the computation and used by the client.

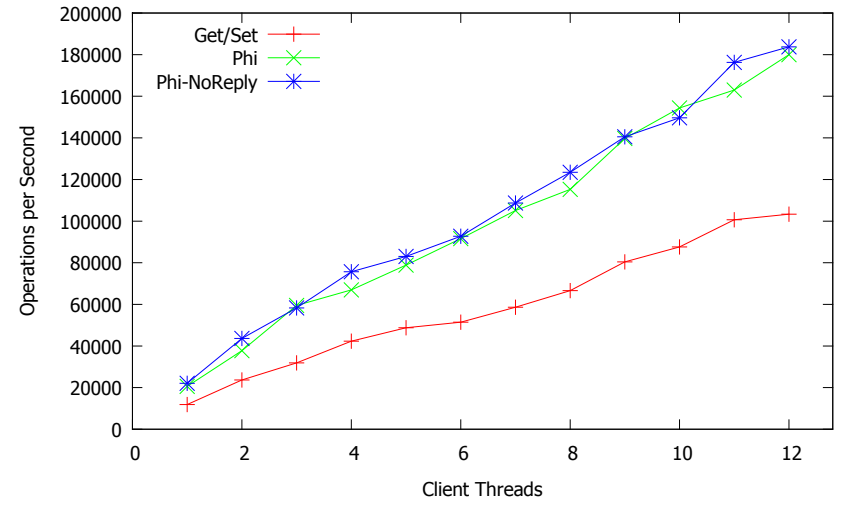

(a) Local, 256 Byte Objects

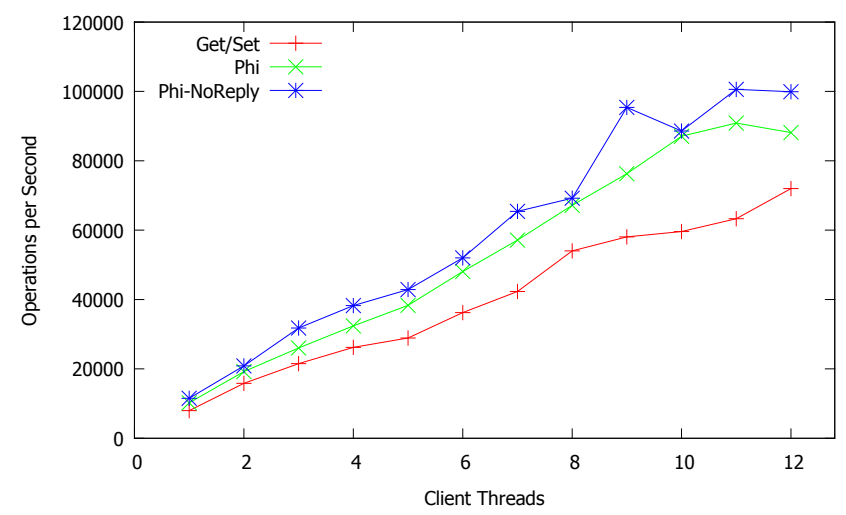

(b) Local, 4K Byte Objects

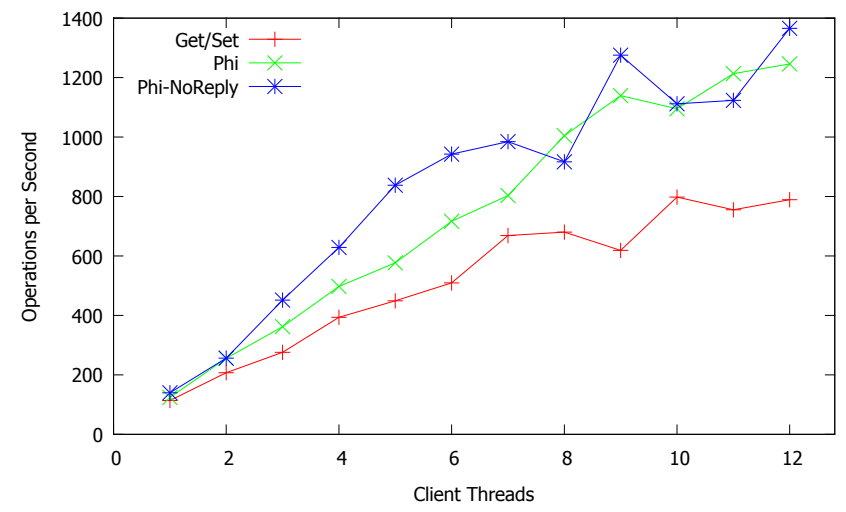

(c) Local, 64K Byte Objects

Figure 2: Micro-benchmark performance for varying object sizes, with the client and server running on the same machine.

Our implementation also does not make any assumptions about the data format. Instead, the invoked function define the type and format of the key's value. Comcast uses protocol buffers, but pickled Python objects, JSON, serialized Java objects, and other formats are possible.

On the other hand, our implementation does assume that the programmer has registered bug-free $\mathrm{C}$ code that are stored in shared objects, and executed directly within the memcached process. Any time code is dynamically loaded into a high-availability process, there are causes for concern. Buggy code can cause the process to crash. Long-running code can keep a thread occupied for an extended period, causing a service degradation or denial of service. 


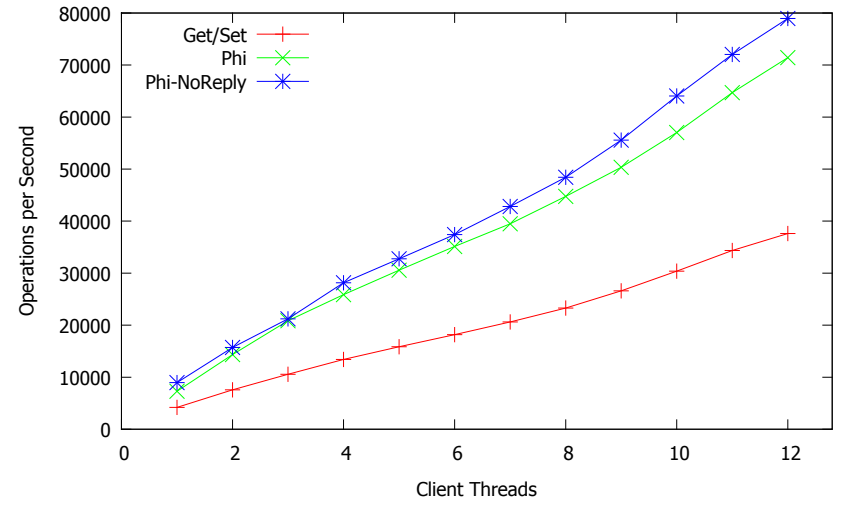

(a) Remote, 256 Byte Objects

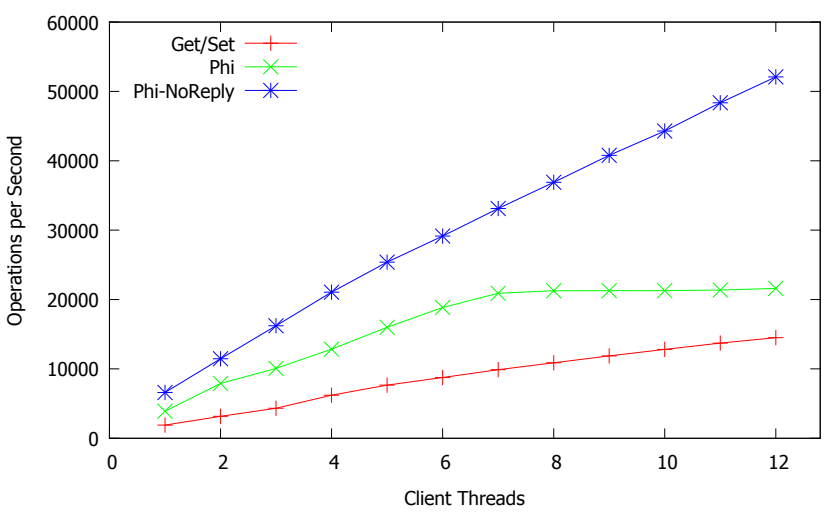

(b) Remote, 4K Byte Objects

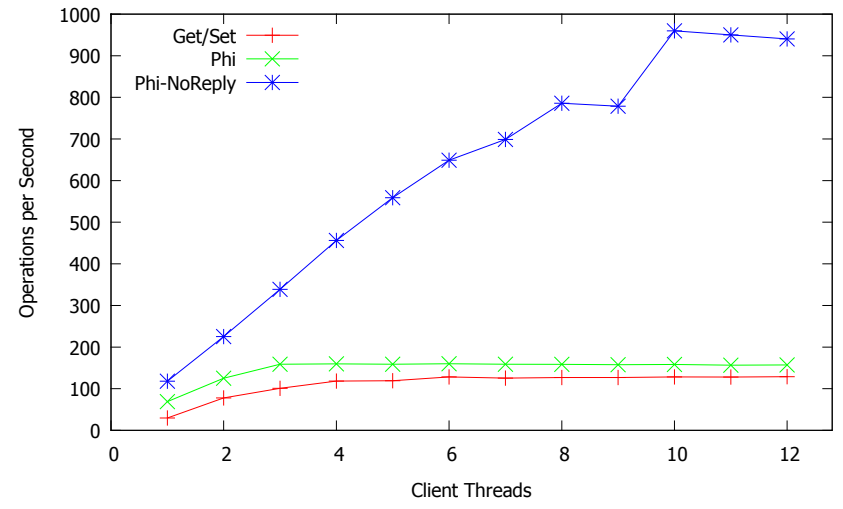

(c) Remote, 64K Byte Objects

Figure 3: Micro-benchmark performance for varying object sizes, with the client and server running on separate machines.

Worse, the code may interact with the operating system to cause the process to block, lose priority, or release needed resources. Our short-term solution is to require system administrator access in order to load code. Low-overhead sandboxing, perhaps via domainspecific languages or IPC between memcached and an interpreter that actually executes the invoked functions, may be better longterm alternatives. However, our focus at this time is to determine whether the lowest-overhead approach provides performance to justify such further investment. Thus we do not currently provide any further sandboxing or reliability guarantees.

\subsection{Sources of Inefficiency}

While our implementation and discussion are focused on memcached, we took care to avoid algorithmic choices specific to memcached. As a result, we incur data copying overheads: When a get is performed, the value is copied from the cache to a temporary buffer. In our system, the function is given this buffer as input. The buffer must be manually reclaimed when invoke returns. Similarly, the function may create a new buffer to store the new value of the key. This buffer is not inserted directly into the cache, but instead is copied into a new pair object, after which it must be reclaimed. The alternative is to hold the lock on a pair throughout the invoke operation. However, this technique fails when the function produces a new value that is of a significantly different size than the input value. In this case, the memcached memory slab allocator would require the new value to be stored in a different slab class.

Likewise, application-specific data format choices introduce overhead. The most obvious example is when the client sends compressed objects to the memcached server: the invoked operation must de-compress before operating on the object, and re-compress afterward. Similar though less expensive overheads accrue in the (common) case where the object format is a serialization of some other format. In addition to another round of copying, this increases CPU utilization. In this paper, we disable compression, but accept copying and serialization overheads as fundamental.

\section{EVALUATION}

To evaluate fetch-and-phi, we ran memcached on a system with two Intel Xeon 5650 chips and 12 GB of RAM. This system has a total of 24 hardware threads (12 cores). We generated requests to this system from an identically configured machine with a single Intel Xeon 5650 (6 cores/12 threads) and 6 GB of RAM. The machines were connected via a switched $1 \mathrm{GBps}$ network. Both machines ran Ubuntu Linux 13.10, GCC 4.8.1, memcached 1.4.20, Oracle Java 1.8.0_11, and spymemcached 2.11.4. All experiments were run five times, and the average is reported. ${ }^{1}$

\subsection{Micro-benchmark Performance}

We populated the memcached server with a set of objects of the same size. Each thread of the client accessed a disjoint set of objects. The workload consists of getting the object, performing an $O(n)$ operation that modifies the object one byte at a time, and then setting the object back into the cache.

Figures 2 and 3 compare the performance of this workload as we vary three parameters. Bars labeled "Get/Set" are the baseline: they correspond to a configuration in which the client gets the object, modifies it, and then sends it back to the server. "Phi" corresponds to the case where the client uses invoke to request that the server perform the operation and then send the new object back to the client. In "Phi-NoReply", the server modifies the object and stores it back to the cache, but only sends an acknowledgment to the client; the new version of the object is not returned.

We ran this experiment at three object sizes: 256 bytes, $4 \mathrm{~K}$ bytes, and $64 \mathrm{~K}$ bytes. We also considered two client configurations. In the first configuration, labeled "remote", we execute the client on a separate machine from the machine running memcached. In the second configuration, labeled "local", we run the client and server on the same machine. This isolates performance improvements that come from reduced network communication.

There are two trends that emerge from this experiment. First, by contrasting the remote and local experiments, we see that the most

\footnotetext{
${ }^{1}$ We conducted additional experiments with three identical clients; this enabled us to saturate the memcached server, but did not reveal new behaviors.
} 
significant savings come from the reduction in round-trip network communication. Using either of the "Phi" approaches, the number of trips is halved. When objects are small, the difference between returning the object, and returning an acknowledgment, is insignificant, and the two "Phi" curves are indistinguishable, though both significantly better than "Get/Set". As object sizes increase, the additional bandwidth savings from sending a simple acknowledgment grows. At the largest object size, halving the number of round-trip communications saves $25 \%$ over "Get/Set".

\subsection{Top-of-the-Hour Performance}

We next explore performance on a simulation that runs a trace from the "top of the hour" problem discussed in Section 1. We collected statistics from a Comcast server over a 10-minute period that included a spike. Every ten seconds, we counted the number of operations performed on each memory slab. The workload exhibited a burst of activity.

Prior to the burst, the workload was $61 \%$ gets and $39 \%$ sets. During the burst, it became $40 \%$ gets, and after the burst, it had 55\% gets. During the burst, there were two primary operations: among small objects, there was an order of magnitude increase in the number of sets, as logic servers marked DVR recordings as started or stopped, and then overwrote a single-recording object of under 1000 bytes. The logic servers typically cached these objects, and thus did not perform a get before the set. However, they still sent the entire object, even though only 4 bytes changed. The second operation was an update to large objects (over 10K bytes). These objects store customers' sets of recordings and their corresponding states. When a small-object set occurs, the large object becomes stale, and must be updated to indicate the new recording state.

Without tracing each individual customer's behavior, we cannot determine the number of sets that (a) were part of a get/set pair, and (b) could be replaced by fetch-and-phi. There is strong evidence that these operations were concentrated on large objects, where the ratio of gets to sets remained relatively constant, though the numbers increased during the burst. Similarly, set operations on small objects can be optimized: rather than sending an updated object, the logic servers can invoke a function that performs the necessary modification to the object, and does not send a reply. Again, we cannot precisely determine the frequency of these operations in the trace. However, they roughly correspond to the increase in sets of small objects during the burst.

We produce workloads of the same shape and operation mix as above via a Java client that uses the spymemcached library to interface to the memcached server. We extended spymemcached to support invoke and register functions. The workload operates on anonymized protocol buffers of either a 480 byte record or a $10 \mathrm{~KB}$ array of records (this is conservative; the trace we captured included arrays up to $200 \mathrm{~KB}$ ). Our client alternates between periods of low activity (a fixed number of operations per second), and bursty periods (execute as many operations as possible). Parameters govern the number of operations that are sets, gets, or invokes, and the object sizes from which randomly selected elements will be chosen for use with those operations. We also control the length and frequency of bursts.

In Figure 4, the cache is populated with $10 \mathrm{~K}$ byte protocol buffers. We oscillated between 30-second periods of burst or non-burst behavior. Each operation is either an unpaired get, or a get/set pair. In this manner, an invoke counts equal to a pair, and captures the notion of an operation from the client's view. In keeping with the workload trace, get operations comprised $44 \%$ of the workload during bursts, and $62 \%$ of the workload during low-utilization periods.

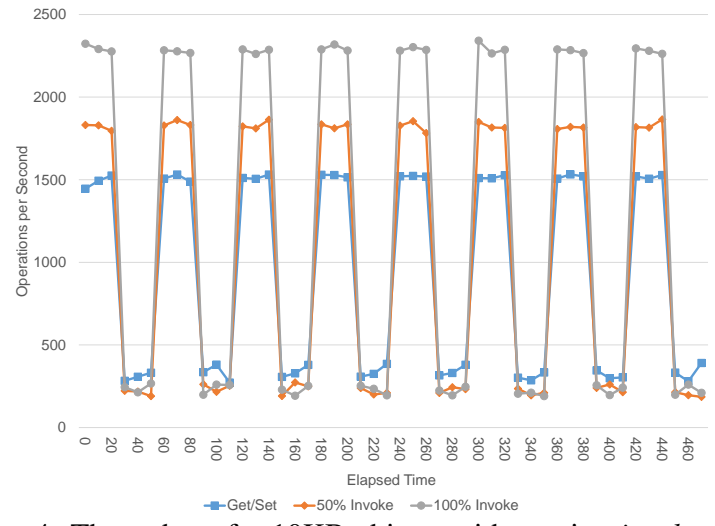

Figure 4: Throughput for $10 \mathrm{~KB}$ objects with varying invoke rates. Get frequency was $44 \%$ during peaks, $62 \%$ otherwise.

This result, which is representative of experiments with varying buffer sizes, shows that even for a modestly large object size, and a computationally expensive operation (while we update on the order of 16 bytes, there is an $O(n)$ overhead to de-serialize the protocol buffer before operating on it, and then another $O(n)$ overhead to serialize it to a byte array before setting it back in the cache), we still achieve a speedup of close to $40 \%$. Furthermore, the benefit is linear in the ratio of get/set pairs that are replaced with invoke operations. The impact on the server is an increase from $21 \%$ to $36 \%$ CPU utilization as Invoke goes from 0 to $100 \%$.

\section{RELATED WORK}

There has been substantial research into distributed key/value stores, both persistent and in-memory. Due to the performancecritical nature of these systems, they are increasingly adopting complex low-level systems techniques to achieve peak performance [9, 4]. In some cases, these systems are also tailored to specific workloads, such as Facebook's TAO [1]. TAO's cache is not a lookaside, but rather a write-through cache, tightly bound to an underlying MySQL-based persistent store. We believe that our work, which studies the performance of fetch-and-phi in an unmanaged language, is complementary to these efforts. In all cases, there is an awareness that decreasing communication bandwidth and lowering the latency of accesses to the cache layer is crucial to overall system performance.

The filtering mechanism we discussed in this paper is similar to several prior proposals $[13,2,12]$. While these generally require the cache to be aware of the object layout, so that they can return specific fields of an object, there is no obstacle preventing such systems from supporting more complex filtering operations. Our work takes the opposite approach, assuming nothing about the object format and leaving it up to the user-provided code to deserialize a copy of the data and compute over it. The best solution for real-world systems and applications is likely to fall somewhere in between: a limited set of operations, but optimized for a workload whose data layout is known by the cache so that copying can be avoided.

The first system we are aware of that supports arbitrary computation directly in the key/value store is Comet [5]. In Comet, objects can either be in an unknown format, or else Lua objects. In the latter case, objects can have triggers attached to them, which execute in response to gets and sets. Our work differs from Comet in a number of regards. From a performance perspective, Comet provides persistence, and hence there is much more room to mask the latency of object serialization and de-serialization. In that re- 
gard, our work can be thought of as providing a lower bound on the best-case latency. More importantly, Comet focused on the security of extensions and the reliability of the overall system. Whereas we studied memcached, which is rarely shared among applications, Comet was intended to support web services with untrusted clients. Thus security of extensions was a more significant factor than in our work.

Other systems which bear relation to our work are OOlong [11] and ZHT [7]. OOlong used the analogy of database triggers to describe a technique for performing computation on a key/value store. OOlong provides some features that are more general than fetch-and-phi, such as allowing the get of object $O$ to cause an update to some other object $K$. Unfortunately, we are not aware of any performance results for this system. Like our work, ZHT allows in-cache computation, but through the less general interface of get/put/delete/append. It also requires the use of protocol buffers, whereas our implementation is agnostic to the data format.

\section{CONCLUSIONS AND FUTURE WORK}

We studied the impact of supporting client-requested computation within the context of a memcached server. Our extensions to memcached, which are based on the concept of a fetch-and-phi operation, have a minimal footprint (under 400 lines of code) and provide an orthogonal mechanism supporting fetch-and-phi and filtering. Our implementation also supports operations that do not send the new object as part of the response. Using traces from Comcast as the basis for our evaluation, we showed that fetch-and-phi operations have the potential to increase throughput by over $30 \%$.

Our success comes at the cost of decreased reliability and programmability. While there are a number of advanced features to eventually explore (e.g., multi-key fetch-and-phi, memcachedspecific optimizations to reduce copying, and low-overhead deserialization), we believe reliability and programmability must be addressed first: In few organizations will performance gains outweigh these concerns. We hope our results encourage new discussions among researchers, and lead to the discovery of new techniques that allow systems to execute code at the most appropriate location, and in the most optimal context, within distributed clouds.

\section{Acknowledgments}

We thank John McCann for many helpful discussions during the conduct of this research. We also thank Sree Kotay and Paul Bosco, who introduced us to the top-of-the-hour problem.

This material is based upon work supported by the National Science Foundation under Grants CAREER-1253362 and CCF-1218530. This work was also supported through a Comcast TechFund grant. Any opinions, findings, and conclusions or recommendations expressed in this material are those of the authors and do not necessarily reflect the views of the National Science Foundation or of Comcast.

\section{REFERENCES}

[1] N. Bronson, Z. Amsden, G. Cabrera, P. Chakka, P. Dimov, H. Ding, J. Ferris, A. Giardullo, S. Kulkarni, H. Li, M. Marchukov, D. Petrov, L. Puzar, Y. J. Song, and V. Venkataramani. TAO: Facebook's Distributed Data Store for the Social Graph. In Proceedings of the USENIX Annual Technical Conference, San Jose, CA, June 2013.

[2] F. Chang, J. Dean, S. Ghemawat, W. Hsieh, D. Wallach, M. Burrows, T. Chandra, A. Fikes, and R. Gruber. Bigtable: A Distributed Storage System for Structured Data. In Proceedings of the 7th Symposium on Operating System Design and Implementation, Seattle, WA, Nov. 2006.
[3] G. DeCandia, D. Hastorun, M. Jampani, G. Kakulapati, A. Lakshman, A. Pilchin, S. Sivasubramanian, P. Vosshall, and W. Vogels. Dynamo: Amazon's Highly Available Key-Value Store. In Proceedings of the 21st ACM Symposium on Operating Systems Principles, Stevenson, WA, Oct. 2007.

[4] A. Dragojevic, D. Narayanan, O. Hodson, and M. Castro. FaRM: Fast Remote Memory. In Proceedings of the 11th USENIX Symposium on Networked Systems Design and Implementation, Seattle, WA, Apr. 2014.

[5] R. Geambasu, A. Levy, T. Kohno, A. Krishnamurthy, and H. Levy. Comet: An Active Distributed Key-Value Store. In Proceedings of the 9th USENIX Symposium on Operating Systems Design and Implementation, Vancouver, BC, Canada, Oct. 2010.

[6] M. Herlihy and N. Shavit. The Art of Multiprocessor Programming. Morgan Kaufmann, 2008.

[7] T. Li, X. Zhou, K. Brandstatter, D. Zhao, K. Wang, A. Rajendran, Z. Zhang, and I. Raicu. ZHT: A Light-Weight Reliable Persistent Dynamic Scalable Zero-Hop Distributed Hash Table. In Proceedings of the 27th International Parallel and Distributed Processing Symposium, Bostom, MA, May 2013.

[8] H. Lim, B. Fan, D. Andersen, and M. Kaminsky. SILT: A Memory-Efficient, High-Performance Key-Value Store. In Proceedings of the 23rd ACM Symposium on Operating Systems Principles, Cascais, Portugal, Oct. 2011.

[9] H. Lim, D. Han, D. Andersen, and M. Kaminsky. MICA: A Holistic Approach to Fast In-Memory Key-Value Storage. In Proceedings of the 11th USENIX Symposium on Networked Systems Design and Implementation, Seattle, WA, Apr. 2014.

[10] memcached.org. Memcached, 2014. http://memcached.org/.

[11] C. Mitchell, R. Power, and J. Li. Oolong: Programming Asynchronous Distributed Applications with Triggers. In Proceedings of the 23rd ACM Symposium on Operating Systems Principles, Cascais, Portugal, Oct. 2011.

[12] S. Patil, M. Polte, K. Ren, W. Tantisiriroj, L. Xiao, J. Lopez, G. Gibson, A. Fuchs, and B. Rinald. YCSB++ : Benchmarking and Performance Debugging Advanced Features in Scalable Table Stores. In Proceedings of the 2nd ACM Symposium on Cloud Computing, Cascais, Portugal, Oct. 2011

[13] The Apache Software Foundation. Apache HBase, 2014. http://hbase.apache.org/. 